\title{
Rapid Method for Electron Tomographic Reconstruction and Three-Dimensional Modeling of the Murine Synapse Using an Automated Fiducial Marker-Free System
}

\author{
Hyun-wook Kim, ${ }^{1}$ Seung Hak Oh, ${ }^{1}$ Namkug Kim, ${ }^{2}$ Eiko Nakazawa, ${ }^{3}$ and Im Joo Rhyu ${ }^{1, *}$ \\ ${ }^{1}$ Department of Anatomy, College of Medicine, Korea University, 126-1 Anam-dong 5ga, Seongbuk-gu, Seoul 136-705, Korea \\ ${ }^{2}$ Department of Radiology, Research Institute of Radiology, Asan Medical Center, University of Ulsan College of Medicine, \\ 388-1 Pungnap-dong, Songpa-gu, Seoul 138-736, Korea \\ ${ }^{3}$ Hitachi High-Technologies Corporation, Nanotechnology Products Business Group, Naka Division, 24-14, Nishi-Shimbashi \\ 1-chome, Minato-ku, Tokyo 105-8717, Japan
}

\begin{abstract}
Electron tomography (ET) has recently afforded new insights into neuronal architecture. However, the tedious process of sample preparation, image acquisition, alignment, back projection, and additional segmentation process of ET repels beginners. We have tried Hitachi's commercial packages integrated with a Hitachi H-7650 TEM to examine the potential of using an automated fiducial-less approach for our own neuroscience research. Semi-thick sections $(200-300 \mathrm{~nm})$ were cut from blocks of fixed mouse (C57BL) cerebellum and prepared for ET. Sets of images were collected automatically as each section was tilted by $2^{\circ}$ increments $\left( \pm 60^{\circ}\right)$. "Virtual" image volumes were computationally reconstructed in three dimension (3D) with the EMIP software using either the commonly used "weighted back-projection" (WBP) method or "topographybased reconstruction" (TBR) algorithm for comparison. Computed tomograms using the TBR were more precisely reconstructed compared with the WBP method. Following reconstruction, the image volumes were imported into the $3 \mathrm{D}$ editing software A-View and segmented according to synaptic organization. The detailed synaptic components were revealed by very thin virtual image slices; 3D models of synapse structure could be constructed efficiently. Overall, this simplified system provided us with a graspable tool for pursuing ET studies in neuroscience.
\end{abstract}

Key words: electron tomography, fiducial, WBP algorithm, TBR algorithm, A-View, 3D reconstruction, synapse

\section{INTRODUCTION}

The development of electron microscopy (EM) to overcome the inherent resolution limits of light for microscopic investigations in the life sciences has played a fundamental role in defining our current understanding of biology by providing high-resolution views of key cellular structures, such as the plasma membrane, cytoskeletal filaments, and organelles, as well as finer structural details such as the inner cristae of mitochondria (Henderson \& Unwin, 1975).

In particular, the transmission electron microscope (TEM) is an extremely useful instrument for obtaining projection images through thin sections cut from biological specimens. However, the density of numerous fine structures and macromolecules that reside within the cytoplasm of any mammalian or plant cell-most of which are readily visible in conventional two-dimensional (2D) micrographs of heavy metal-stained, thin sectioned material—means that not only does the spatial/structural information for these structures overlap in each projection image (i.e., relative to the depth of the section), but shows different morphologies according to projection angles (Baumeister et al., 1999). To solve these problems, electron tomography (ET) techniques have been applied by leading groups in the field over the past 20 years or so, but have been used more widely within

() MICROSCOPY SOCIETY OF AMERICA 2013

${ }^{*}$ Corresponding author. E-mail: irhyu@korea.ac.kr the cell biology community over the past decade and have contributed substantially to more advanced morphological studies of cells and tissues in 3D configuration (McIntosh et al., 2005). On the other hand, serial section TEM techniques have also been used for the ultrastructure-based morphological study of large biological samples before the development of ET (Lee et al., 2005).

ET methods were used to successfully elucidate the process by which human and simian immunodeficiency viruses infect T-cells of the immune system (Sougrat et al., 2007), and to clearly capture and visualize (in 3D) the finer structure of mitochondria, as well as networks of actin filaments (Urban et al., 2010).

The ET has contributed remarkably to the advance of neuroscience research. Several ET studies have characterized the morphometric features of synaptic structures at high resolution, such as number and size of synaptic vesicles (SVs), the area of presynaptic density, and the architecture of the synaptic cleft (SC) distance (Lee et al., 2005; Siksou et al., 2007). Such data have provided much more reliable insights into the organization and structural composition of neuronal cells/tissues when compared with conventional 2D micrographs/projection images.

Typically, ET studies are composed of four steps: (1) acquisition of serially tilted images (i.e., a "tilt series"), (2) precise, digital alignment of adjacent images based on the trajectories of fiduciary gold particles adhered to the 
sections before imaging begins, (3) computed reconstruction of an image volume from the aligned images to yield a "virtual block," typically using back-projection algorithms, and (4) generation and analysis of 1-pixel-thick virtual image slices. Furthermore, painstaking 3D modeling of the resulted image volume is often used to address particular research questions. Overall, these steps tend to be both complicated and time consuming for a layman, thus serving as major obstacles for beginners in the field.

Recently, several semi automated and/or automated software systems have been developed by different companies and academic groups around the world in order to remove such barriers and to increase the use of ET for general research. Furthermore, many scientists discussed of TEM automation for the application to ET technique, and several companies applied these theories to automate system commercialization (Zheng et al., 2004; Nickell et al., 2005; Suloway et al., 2009). The various programs for automated ET system were proposed and developed such as Leginon (Suloway et al., 2009), UCSF Tomo (Zheng et al., 2004), TOM (Nickell et al., 2005), and Xplore3D (FEI Company), based on different theories for perfect data summarizing. Cao and colleagues proposed an automatic method for detecting and tracking fiducial makers for alignment and adapted IMOD software, and another group suggested membrane system-related tomography system via IMOD software package (Donohoe et al., 2006; Cao et al., 2010).

In this report, we present our inaugural results using one such example of a commercial software system for automated ET- the software package Electron Tomography Image Processing (EMIP) from Hitachi High Technologies (Hitachi High-Technologies Corporation, Japan) installed on the Hitachi H-7650 TEM (Hitachi High-Technologies Corporation). The EMIP system comprises an automatic image capturing function for tilt series acquisition, as well as $3 \mathrm{D}$ reconstruction programs within the software package. The advantage of this system is that it does not require fiducial gold particles and manual alignment of serially tilted images. In addition to this software, we applied the software A-View (developed at Radiology Department in Asan Medical Center, Korea), which was originally developed as a volume editing program for diagnostic imaging [e.g., magnetic resonance imaging (MRI) and computed tomography (CT)]. In this study, we present a useful and accessible strategy for undertaking ET studies within a broader research program by combining an automated "fiducial marker-free" system with readily available 3D volume editing software.

\section{Materials and Methods}

\section{Preparation of Biological Samples for EM}

Forty-day-old C57BL mice were used in this study. After intraperitoneal injection of sodium pentobarbital $(50 \mathrm{mg} /$ $\mathrm{kg}$ ), mice were perfused transcardially with $0.9 \%$ saline followed by fixatives ( $1 \%$ paraformaldehyde, $1 \%$ glutaraldehyde in $0.2 \mathrm{M}$ phosphate buffer). After the brain was re- moved from the skull, the cerebellum was dissected and sections were stored in the same fresh fixative overnight at $4^{\circ} \mathrm{C}$. Fixed cerebellar sections were washed with phosphate buffer several times and then post-fixed in $2 \%$ osmium tetroxide for $2 \mathrm{~h}$. En bloc staining was carried out using 2\% uranyl acetate in $50 \%$ ethanol for $24 \mathrm{~h}$ followed by embedding in Epon-Araldite resin using a Reichert-Jung Ultracut-E microtome. Plastic sections with three different thicknesses $(200,250$, or $300 \mathrm{~nm})$ were cut. Each section was mounted on formvar-coated mesh grids and stained with $2 \%$ uranyl acetate for 20 min followed by Reynold's lead citrate for $10 \mathrm{~min}$. After preliminary survey of the three different section thicknesses using a $120 \mathrm{kV}$ TEM, we decided that a thickness of $250 \mathrm{~nm}$ was most appropriate for subsequent imaging and reconstruction by ET.

\section{Automatic Tomography and 3D Reconstruction of "Virtual Blocks"}

Grids holding $250 \mathrm{~nm}$ sections were loaded into a specimen holder that tilts automatically by $2^{\circ}$ steps from $+60^{\circ}$ to $-60^{\circ}$. Each tilt series of a region of interest (images were acquired after an automatic focus step at each tilt angle) collected using the Hitachi H-7650 TEM took 35-40 min to acquire automatically using this protocol. After tilt series data sets were loaded into the EMIP software, 3D reconstruction was carried out using two different kinds of backprojection algorithms [topography-based reconstruction (TBR); weighted back-projection (WBP)]. Virtual slices along the $Z$-axis were computed and then exported into 16-bit TIFF format.

\section{D Reconstruction}

Serial Z-slice images were imported into A-View software ready for further analysis. Each synaptic component was segmented using editing tools within the A-View program by segmenting each component on every section in order to render and visualize the synaptic vesicle membrane, synaptic vesicle lumen, synaptic vesicle connection, presynaptic membrane, postsynaptic membrane, SC, and postsynaptic density clearly in 3D. Pilot morphometric measurements were performed to check the potential application of this approach for further studies using the A-View program.

\section{Results}

Serially tilted images were successfully captured from $+60^{\circ}$ to $-60^{\circ}$. A comparison of two different views of the same section acquired at $0^{\circ}$ tilt angle versus $+60^{\circ}$ tilt (data not shown due to limited space) revealed that at high tilt the same synapse appears very dark and looks to be altogether different, although it is the same synapse observed at $0^{\circ}$. However, we were able to successfully capture complete tilt series data sets for ET in $<40$ min, due to automated tilting using eucentric control and focusing.

A comparison of the virtual blocks computed in EMIP using either the WBP or TBR algorithms (Figs. 1A, 1B, respectively) suggested that the quality of $3 \mathrm{D}$ image recon- 


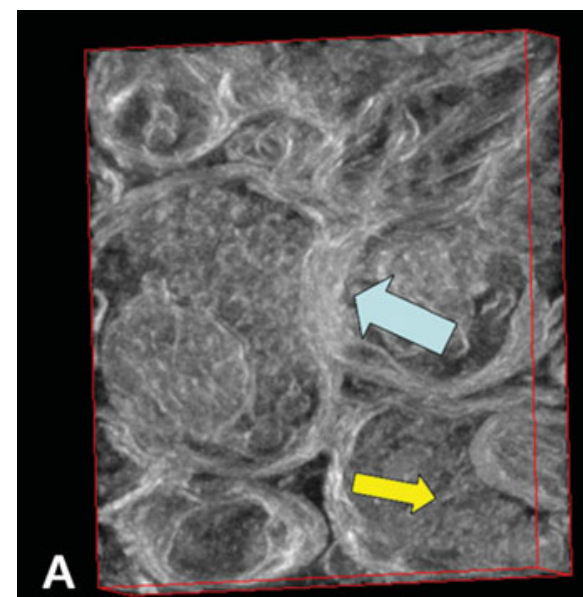

WBP

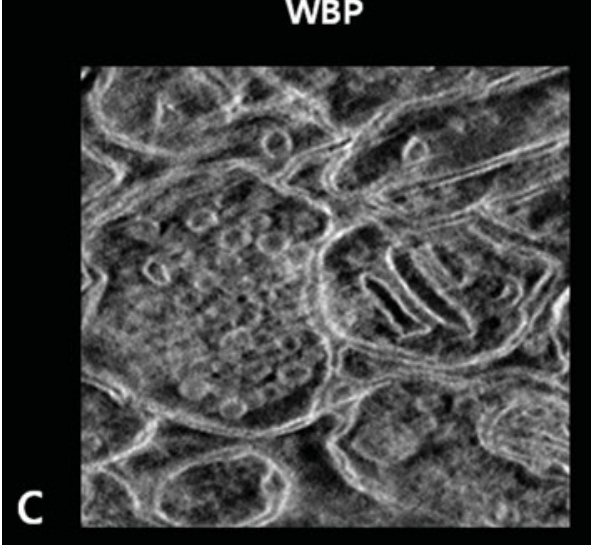

D

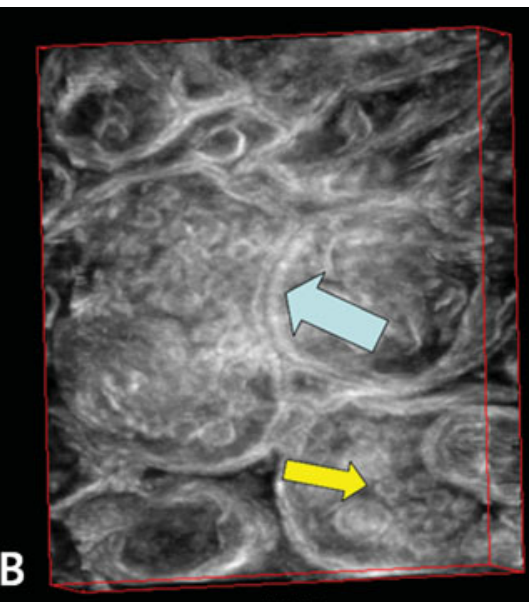

TBR

struction using TBR was more appropriate for our analysis of the presynaptic membrane (large arrow) and SV (small arrow). The virtual slice image obtained from WBP revealed detailed images of presynaptic and postsynaptic components, but background noise appeared to lower the final image quality (Fig. 1C); virtual slices obtained from the TBR block provided clear visualization of the same synaptic structures because of reduced background noise (Fig. 1D). However, in terms of speed of reconstruction, WBP was faster than TBR (the time for TBR processing was $\sim 140 \mathrm{~min}$ when it was operated at parallel dual-core system compared with 30 min for WBP processing).

Serial virtual slices along the $Z$-axis produced by EMIP were imported into A-View program and calibrated for exact image processing and morphometric measurements (pixel size: $1.458 \mathrm{~nm}$; serial view, sagittal view, coronal view, and reconstructed 3D model).

The voxel size of virtual slice was $1.458 \times 1.458 \times 1.458$ $\mathrm{nm}^{3}$ for images taken at $12,000 \times$ magnification. We were able to identify detailed structures of the synapse such as the SVs and fine filamentous structures that connect SVs within $1.458 \mathrm{~nm}$ thickness (Fig. 2b), which is not easy to identify with conventional TEM. The SV structures were divided into the vesicular lumen and its content on the virtual slice, and the omega profiles of SVs docking to the presynaptic terminal were observed (Figs. 2C-2E).

The segmented structures on each slice were stacked, and 3D models of synapse were generated by surface render-

ing. The 3D configurations of presynaptic terminal, postsynaptic terminal, SV, and postsynaptic density are demonstrated in this model (Fig. 3A) and some morphometric data are also available (Fig. 3B).

\section{Discussion}

ET provides two major advantages: (1) ET affords examination and detailed analysis of virtual slices at nanometerscale thicknesses, which is not possible with physical sectioning, (2) 3D information of the tissue is available by using relatively thick sections up to 250-300 $\mathrm{nm}$ using a $120 \mathrm{kV}$ conventional TEM (Baumeister et al., 1999; Siksou et al., 2007). The contribution of ET in the neuroscience research has been significant, especially for detailed 3D characterization of synaptic structures including SV (Shoop et al., 2002). Recently, important proteins were mapped in details, and a model for protein composition of the postsynaptic density was also proposed (Chen et al., 2008). Nevertheless, there are little publications using ET, because its experimental step is complicated and time consuming. We combined simplified automatic acquisition system and easy 3D image processing system, which expects to lower the entrance barrier to ET research. The automated tilting, focusing, and capturing image shortened the time needed for image acquisition.

In this experiment, manual alignment step using fiducial particles was skipped, which is one of the difficult steps 

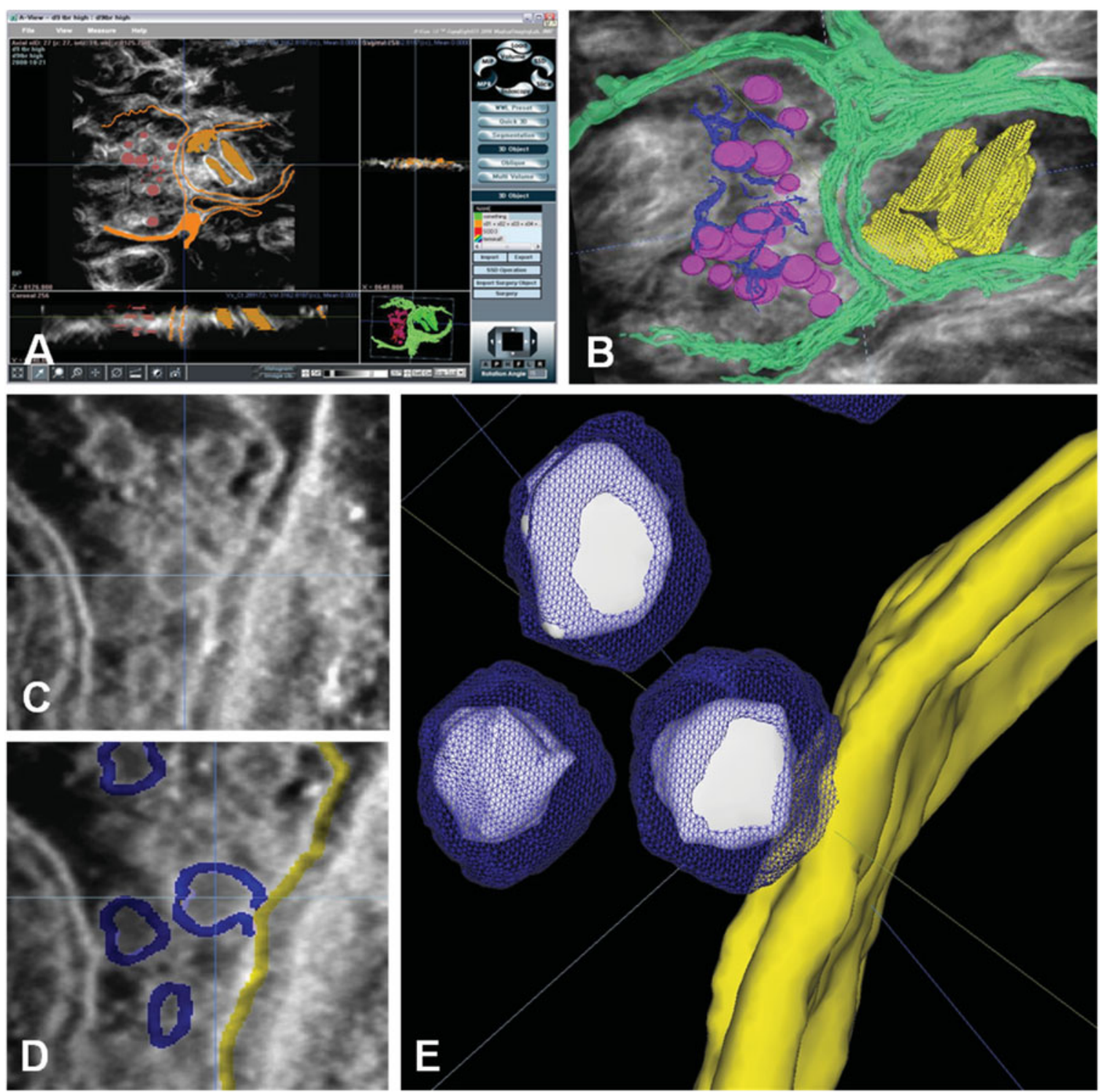

Figure 2. Representative view of three-dimensional (3D) modeling synapse. The editing status can be checked in three planes (A). The fine filaments linking synaptic vesicles are clearly observed (B): SV, purple; SVC, blue; pre- and postsynaptic membrane, green; ER in postsynaptic terminal, yellow. The fusion of synaptic vesicle and presynaptic membrane shows typical omega-shaped morphology $(\mathbf{C})$. The 3D modeling of synaptic vesicle fused with presynaptic terminal is demonstrated after segmentation of synaptic vesicles and presynaptic membrane (D, E): SVM, blue; SVL, white; Pre, yellow. SV, synaptic vesicles; SVC, synaptic vesicle connection; SVM, synaptic vesicle membrane; SVL, synaptic vesicle lumen; Pre, presynaptic membrane.

of ET. Previous studies reported automation or fiducial maker-free system algorithms for time-reducing electron microscopic tomography studies (Liu et al., 1995; Zheng et al., 2004; Nickell et al., 2005; Baba \& Katayama, 2008; Suloway et al., 2009). The automated fiducial gold particle free system might be more useful to map multiple proteins by immune-gold labeling by avoiding gold particles for alignment.

As we expected, we could obtain a series of tilted image fast, and virtual block was constructed by back projection within a couple of hours, which would be shortened with advance of computer CPU speed. In order to obtain fast rough topographic data of region of interests, the virtual slice can be obtained within $1 \mathrm{~h}$ when WBP algorithm is applied. If high-quality image data are needed, TBR could be a choice, although it takes more than $3 \mathrm{~h}$. TBR uses unique algorithms to minimize the "missing wedge effect" (Baba \& Katayama, 2008), which is a long problem of ET due to limitation of tilting angle. To overcome the "missing wedge effect," double tilting series were used for reconstruction, and home-made $360^{\circ}$ tilting stage was applied (Penczek et al., 1995).

The virtual volume should be analyzed at the level of $3 \mathrm{D}$ virtual slices to analyze and demonstrate scientific fact. 3D editing programs such as 3DS-Max, Maya, Amira, and IMOD are popular rendering programs for ET reconstruc- 

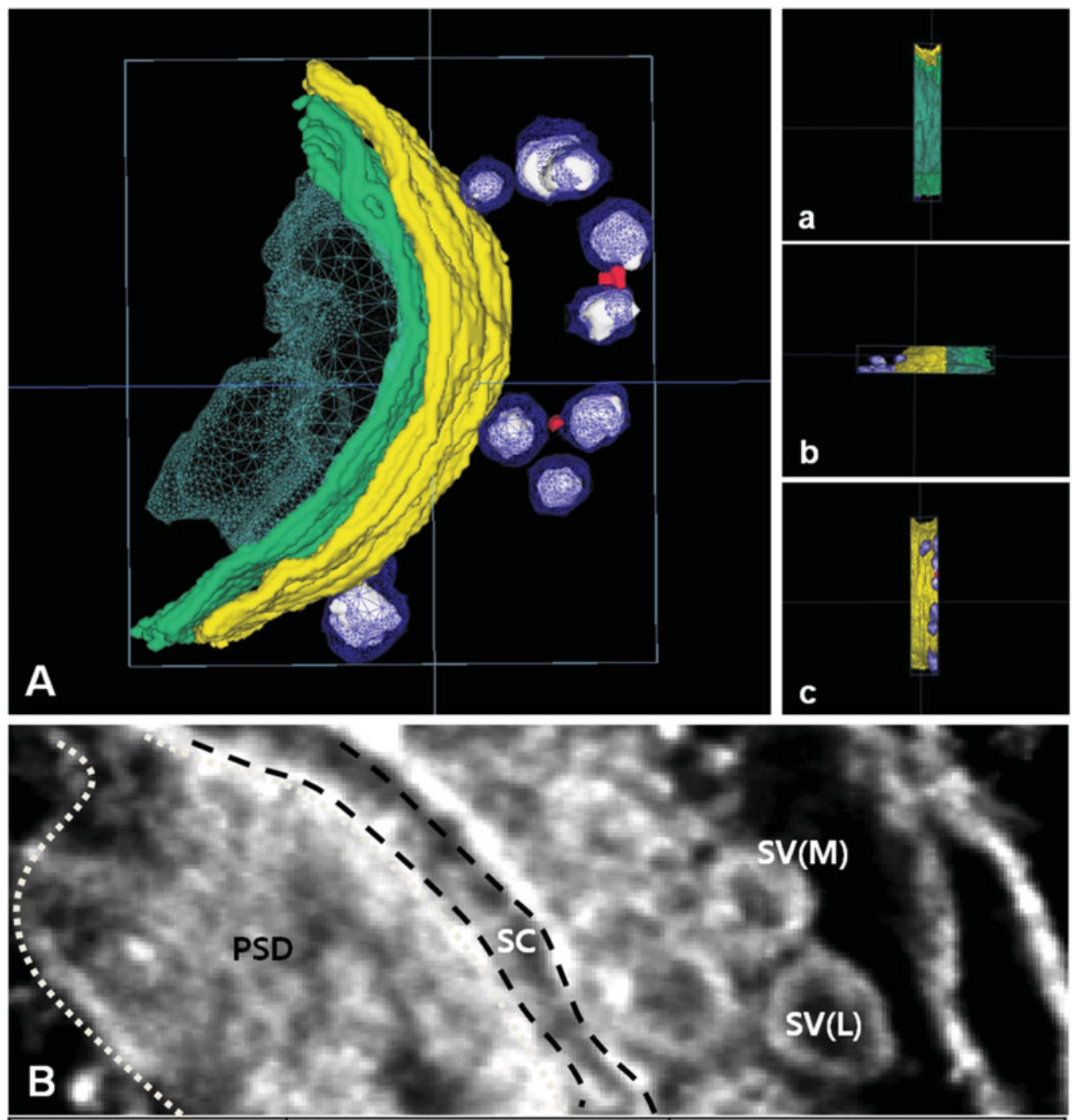

\begin{tabular}{|c|c|c|}
\hline Contents & Length $(\mathrm{nm})$ & Volume $\left(\mathrm{nm}^{3}\right)$ \\
\hline $\mathrm{SV}(\mathrm{L})(\mathrm{n}=20)$ & 30.02 (diameter) \pm 1.29 & $15523.69 \pm 660.8$ \\
\hline $\mathrm{SV}(M),(\mathrm{n}=20)$ & $15.64 \pm 0.47$ & $24763.85 \pm 1056.9$ \\
\hline $\mathrm{SV}(\mathrm{L}+\mathrm{M}),(\mathrm{n}=20)$ & $45.66 \pm 1.23$ & $40287.55 \pm 1506.2$ \\
\hline $\mathrm{PSD},(\mathrm{n}=4)$ & $\mathrm{n} / \mathrm{a}$ & $1174838.677 \pm 108281$ \\
\hline $\mathrm{SC},(\mathrm{n}=20)$ & $18.3 \pm 0.75$ & $\mathrm{n} / \mathrm{a}$ \\
\hline
\end{tabular}

Figure 3. Morphometric measurement of synaptic components was performed after three-dimensional (3D) modeling (A) using A-View program. SVM, blue; SVL, white; SVC, red; Pre, yellow; Post, green; PSD, cyan. Other angle view of 3D model (a: left-side view, b: top view, and c: right-side view). Synaptic components were segmented for measurement (B). Table shows the measured value of each component (mean \pm SD from each numbers of rendering). SVM, synaptic vesicle membrane; SVL, synaptic vesicle lumen; SVC, synaptic vesicle connection; Pre, presynaptic membrane; Post, postsynaptic membrane; PSD, post synaptic density.

tion (Kremer et al., 1996; Morone et al., 2006). Especially IMOD software has the highest level of popularity on ET field for reconstruction, as it is free for users and provides supports from University of Colorado (http://bio3d .colorado.edu/imod/). In this study, we used "A-View program," which is originally developed for 3D analysis of CT and MRI analysis, which is operated under the Windows ${ }^{\circledR}$ system (Microsoft). A-View provides a simple and userfriendly interface to segment regions of interest and model them with ease (Park et al., 2006). We can measure some morphometric parameters such as length, area, and volume in the given environment in 3D. The morphometric characteristics of murine synapse collected in this study were matched with previous research with EM. We could also 
measure whole SV volume and the volume of its contents separately, which should be confirmed by stereological approaches.

In this study, we suggest a new combination of automated tomography system and efficient $3 \mathrm{D}$ volume rendering with image analysis system (A-View program), which would introduce many biological scientists to run ET researches more conveniently.

\section{ACKNOWLEDGMENT}

This work was supported by Basic Science Research Program through the National Research Foundation (NRF) funded by the Ministry of Education, Science and Technology (2010-0012753).

\section{References}

Baba, N. \& Katayama, E. (2008). A novel "ghost"-free tomographic image reconstruction method applicable to rotaryshadowed replica specimens. Ultramicroscopy 108(3), 239-255.

Baumeister, W., Grimm, R. \& Walz, J. (1999). Electron tomography of molecules and cells. Trends Cell Biol 9(2), 81-85.

CaO, M., Takaoka, A., Zhang, H.B. \& Nishi, R. (2010). An automatic method of detecting and tracking fiducial markers for alignment in electron tomography. J Electron Microsc (Tokyo) 60(1), 39-46.

Chen, X., Winters, C.A. \& Reese, T.S. (2008). Life inside a thin section: Tomography. J Neurosci 28(38), 9321-9327.

Donohoe, B.S., Mogelsvang, S. \& Staehelin, L.A. (2006). Electron tomography of ER, golgi and related membrane systems. Methods 39(2), 154-162.

Henderson, R. \& Unwin, P.N. (1975). Three-dimensional model of purple membrane obtained by electron microscopy. Nature 257(5521), 28-32.

Kremer, J.R., Mastronarde, D.N. \& McIntosh, J.R. (1996). Computer visualization of three-dimensional image data using IMOD. J Struct Biol 116(1), 71-76.

Lee, K.J., PARK, C.H. \& Rhyu, I.J. (2005). Efficient three-dimensional reconstruction of synapse with high-voltage electron microscopy. J Electron Microsc (Tokyo) 54(2), 139-141.

Liu, Y., Penczek, P.A., McEwen, B.F. \& Frank, J. (1995). A marker-free alignment method for electron tomography. Ultramicroscopy 58(3-4), 393-402.
McIntosh, R., Nicastro, D. \& Mastronarde, D. (2005). New views of cells in 3D: An introduction to electron tomography. Trends Cell Biol 15(1), 43-51.

Morone, N., Fujimara, T., Murase, K., Kasai, R.S., Ike, H., Yuasa, S., Usukura, J. \& Kusumi, A. (2006). Three-dimensional reconstruction of the membrane skeleton at the plasma membrane interface by electron tomography. J Cell Biol 174(6), 851-862.

Nickell, S., Forster, F., Linaroudis, A., Net, W.D., Beck, F., Hegerl, R., Baumeister, W. \& Plitzko, J.M. (2005). TOM software toolbox: Acquisition and analysis for electron tomography. J Struct Biol 149(3), 227-234.

Park, I.S., Han, J.W., Lee, K.J., Lee, N.J., Lee, W.T., Park, K.A. \& Rhyu, I.J. (2006). Evaluation of morphological plasticity in the cerebella of basketball players with MRI. J Kor Med Sci 21(2), 342-346.

Penczek, P., Marko, M., Buttle, K. \& Frank, J. (1995). Doubletilt electron tomography. Ultramicroscopy 60(3), 393-410.

Shoop, R.D., Esquenazi, E., Yamada, N., Ellisman, M.H. \& BERG, D.K. (2002). Ultrastructure of a somatic spine mat for nicotinic signaling in neurons. J Neurosci 22(3), 748-756.

Siksou, L., Rostaing, P., Lechaire, J.P., Boudier, T., Ohtsuka, T., Fejtova, A., Kao, H.T., Greengard, P., Gundelfinger, E.D., Triller, A. \& Marty, S. (2007). Three-dimensional architecture of presynaptic terminal cytomatrix. J Neurosci 27(26), 6868-6877.

Sougrat, R., Bartesaghi, A., Lifson, J.D., Bennett, A.E., Bess, J.W., Zabransky, D.J. \& Subramaniam, S. (2007). Electron tomography of the contact between $\mathrm{T}$ cells and SIV/HIV-1: Implications for viral entry. PLoS Pathog 3(5), e63.

Suloway, C., Shi, J., Cheng, A., Pulokas, J., Carragher, B., Potter, C.S., Zheng, S.Q., Agard, D.A. \& Jensen, G.J. (2009). Fully automated, sequential tilt-series acquisition with Leginon. J Struct Biol 167 (1), 11-18.

Urban, E., Jacob, S., Nemethova, M., Resch, G.P. \& Small, J.V. (2010). Electron tomography reveals unbranched networks of actin filaments in lamellipodia. Nat Cell Biol 12 (5), 429-435.

Zheng, Q.S., Braunfeld, M.B., Sedat, J.W. \& Agard, D.A. (2004). An improved strategy for automated electron microscopic tomography. J Struct Biol 147(2), 91-101. 